\title{
Efficient diffuse auroral electron scattering by electrostatic electron cyclotron harmonic waves in the outer magnetosphere: A detailed case study
}

\author{
Binbin Ni, ${ }^{1}$ Jun Liang, ${ }^{2}$ Richard M. Thorne, ${ }^{1}$ Vassilis Angelopoulos,,${ }^{3,4}$ Richard B. Horne, ${ }^{5}$ \\ Marina Kubyshkina, ${ }^{6}$ Emma Spanswick, ${ }^{2}$ Eric F. Donovan, ${ }^{2}$ and Dirk Lummerzheim ${ }^{7}$ \\ Received 23 August 2011; revised 13 October 2011; accepted 18 November 2011; published 31 January 2012.
}

[1] This paper is a companion to a paper by Liang et al. (2011) which reports a causal connection between the intensification of electrostatic ECH waves and the postmidnight diffuse auroral activity in the absence of whistler mode chorus waves at $\mathrm{L}=11.5$ on the basis of simultaneous observations from THEMIS spacecraft and NORSTAR optical instruments during 8-9 UT on February 5, 2009. In this paper, we use the THEMIS particle and wave measurements together with the magnetically conjugate auroral observations for this event to illustrate an example where electrostatic electron cyclotron harmonic $(\mathrm{ECH})$ waves are the main contributor to the diffuse auroral precipitation. We use the wave and particle data to perform a comprehensive theoretical and numerical analysis of ECH wave driven resonant scattering rates. We find that the observed $\mathrm{ECH}$ wave activity can cause intense pitch angle scattering of plasma sheet electrons between $100 \mathrm{eV}$ and $5 \mathrm{keV}$ at a rate of $>10^{-4} \mathrm{~s}^{-1}$ for equatorial pitch angles $\alpha_{e q}<30^{\circ}$. The scattering approaches the strong diffusion limit in the realistic ambient magnetic field to produce efficient precipitation loss of $<\sim 5 \mathrm{keV}$ electrons on a timescale of a few hours or less. Using the electron differential energy flux inside the loss cone estimated based upon the energy-dependent efficiency of ECH wave scattering for an 8-s interval with high resolution wave data available, the auroral electron transport model developed by Lummerzheim (1987) produced an intensity of $\sim 2.3 \mathrm{kR}$ for the green-line diffuse aurora. Separately, Maxwellian fitting to the electron differential flux spectrum produced a green-line auroral intensity of $\sim 2.6 \mathrm{kR}$. This is in good agreement with the $\sim 2.4 \mathrm{kR}$ green-line auroral intensity observed simultaneously at the magnetic foot point (as inferred using the event-adaptive model of Kubyshkina et al. $(2009,2011)$ ) of the location where the in situ observations were obtained. Our results support the scenario that enhanced ECH emissions in the central plasma sheet (CPS) can be an important or even dominant driver of diffuse auroral precipitation in the outer magnetosphere. This paper is an important compliment to recent work that has shown lower band and upper band chorus to be mainly responsible for the occurrences of diffuse aurora in the inner magnetosphere.

Citation: Ni, B., J. Liang, R. M. Thorne, V. Angelopoulos, R. B. Horne, M. Kubyshkina, E. Spanswick, E. F. Donovan, and D. Lummerzheim (2012), Efficient diffuse auroral electron scattering by electrostatic electron cyclotron harmonic waves in the outer magnetosphere: A detailed case study, J. Geophys. Res., 117, A01218, doi:10.1029/2011JA017095.

\section{Introduction}

[2] Based on a recent survey of 11 years of particle data from the DMSP satellites, Newell et al. [2009] showed that the diffuse aurora dominantly contains $84 \%$ of the energy flux into the ionosphere during low solar wind driving

\footnotetext{
${ }^{1}$ Department of Atmospheric and Oceanic Sciences, University of California, Los Angeles, California, USA.

${ }^{2}$ Department of Physics and Astronomy, University of Calgary, Calgary, Alberta, Canada.

Copyright 2012 by the American Geophysical Union. 0148-0227/12/2011JA017095
}

conditions and $71 \%$ during high solar wind driving conditions. Although both ions and electrons precipitate in the diffuse aurora, the average integral number flux of the precipitating auroral ions is typically 1 to 2 orders of magnitude

\footnotetext{
${ }^{3}$ Institute of Geophysics and Planetary Physics, University of California, Los Angeles, California, USA.

${ }^{4}$ Department of Earth and Space Sciences, University of California, Los Angeles, California, USA.

${ }^{5}$ British Antarctic Survey, Cambridge, UK.

${ }^{6}$ Institute of Physics, Saint Petersburg State University, St. Petersburg, Russia.

${ }^{7}$ Geophysical Institute, University of Alaska Fairbanks, Fairbanks, Alaska, USA.
} 
less than that of the precipitating auroral electrons [Hardy et al., 1985, 1989]. The diffuse auroral region extends over a latitude range of less than $5^{\circ}$ to $10^{\circ}$ or more and maps along magnetic field lines from the outer radiation belts to the entire central plasma sheet (CPS). The diffuse auroral precipitation boundaries and peak location are strongly dependence on solar wind condition. Due to the eastward drift of electrons from nightside CPS, the diffuse aurora is most intense during midnight and morning hours and often relatively insignificant from postnoon through dusk MLT sector.

[3] It is generally accepted that the source population for the diffuse aurora originates from CPS electrons of $10 \mathrm{seV}$ to several keV [Lui et al., 1977], whose precipitation losses due to resonant wave-particle interactions account for the occurrence of the diffuse aurora [e.g., Swift, 1981; Fontaine and Blanc, 1983; Coroniti, 1985]. However, the relative roles of the two main candidates, electrostatic electron cyclotron harmonic (ECH) waves and whistler mode chorus waves, in driving the diffuse auroral precipitation have remained controversial for over four decades [e.g., Kennel et al., 1970; Lyons, 1974; Horne et al., 1981; Belmont et al., 1983; Roeder and Koons, 1989; Johnstone et al., 1993; Meredith et al., 2000; Horne and Thorne, 2000; Horne et al., 2003; Ni et al., 2008]. A CRRES survey of ECH waves and upperband chorus by Meredith et al. [2009] indicated that the global morphology of the two wave modes and the particles, together with their dependence on geomagnetic activity, is reminiscent of the global morphology of the diffuse aurora. Until recently, comprehensive theoretical and modeling studies combined with CRRES observations [Thorne et al., 2010; Ni et al., 2011a, 2011b] have revealed that scattering by chorus is the dominant cause of the most intense diffuse auroral precipitation on the nightside in the inner magnetosphere $\left(<\sim 8 \mathrm{R}_{\mathrm{e}}\right)$. However, the mechanism(s) for diffuse auroral precipitation at higher L-shell regions remains unclear. Based on a global survey of chorus waves using THEMIS observations, Li et al. [2009a] showed that chorus emissions are as weak as below a few pT beyond $\sim 8 \mathrm{R}_{\mathrm{E}}$ in the midnight-to-dawn MLT sector, insufficient to cause efficient diffuse auroral precipitation. In contrast, moderately strong ECH emissions $(>0.1 \mathrm{mV} / \mathrm{m})$ have been reported to extend to L $\geq 10$ [Roeder and Koons, 1989; Ni et al., 2011c]. Therefore, ECH waves can be potentially important for understanding the origin of the diffuse aurora at higher invariant latitudes and the evolution of outer CPS electron distribution.

[4] To investigate the diffuse aurora and its conjunction to plasma wave activity in the outer CPS, Liang et al. [2011, hereinafter LJ2011] performed a detailed event study using the simultaneous in situ wave and particle observations by a cluster of three THEMIS [Angelopoulos, 2008] inner probes (THA, THD and THE) and ground optical auroral observations from the NORSTAR MultiSpectral Imager (MSI) and Meridian Scanning Photometer (MSP) operated at Gillam (GILL) Canada during 08-09 UT on 5 February 2009. Using the event-adaptive, time-dependent magnetic field model of Kubyshkina et al. [2009, 2011], and comparing the model magnetic fields with the THEMIS observed magnetic fields, a credible magnetosphere-ionosphere mapping in the CPS at $\mathrm{L}=11.5$ has been obtained by LJ2011 in order to estimate the satellite footprints. Those ionospheric footprints were found to be located well within the field-of-view (FOV) of the GILL MSI and MSP during the event interval of interest. LJ2011's analyses of the THEMIS FilterBank (FBK) and FFT wave data showed either an absence of chorus activity or very weak chorus activity and captured an unambiguous positive correlation between the simultaneously observed intensities of the diffuse auroral precipitation and $\mathrm{ECH}$ emissions. To validate their proposed scenario that $\mathrm{ECH}$ wave scattering was responsible for the NORSTAR MSP observed green-line auroral intensities, LJ2011 assessed the precipitating energy fluxes under a rough assumption that the loss cone is fully filled for all electron energies. Their estimate was qualitatively comparable to but larger than the actually observed values, which is expected and suggests that a more careful quantitative examination of $\mathrm{ECH}$ wave scattering effect should be implemented to better understand the favorable connection between ECH waves and the diffuse auroral intensity in the outer magnetosphere established in their case study.

[5] The present work is a companion paper to LJ2011's paper, focusing on a comprehensive theoretical and numerical analysis of the role of ECH emissions in scattering the CPS electrons and driving the postmidnight diffuse auroral precipitation at $\mathrm{L}=11.5$ reported by $\mathrm{LJ} 2011$. This paper is organized as follows: in section 2 we describe the performance of various magnetic field models. We also establish the ECH wave model of wave intensity frequency distribution using the THEMIS EFI [Bonnell et al., 2008] observations and of latitudinal variation of wave normal angle distribution using the HOTRAY [Horne, 1989] simulations. Based on quasi-linear theory, section 3 presents ECH wave scattering rates of plasma sheet electrons obtained using the Kubyshkina et al. [2011] event-adaptive magnetic field model and the wave model described in section 2. Section 4 performs a quantitative analysis of electron precipitation flux based upon diffusion rate results, which is subsequently compared with the NORSTAR GILL MSP observations to understand the exact role of ECH wave driven diffusion in influencing the diffuse auroral intensity. We discuss our results and conclude the paper in section 5 .

\section{Magnetic Field Models and ECH Wave Model}

[6] As pointed out above, the event of interest occurred during 08-09 UT on 5 February 2009. Here we further concentrate on a wave burst mode period 08:38:43-08:38:51 UT on THD when the probe was located very close the magnetic equator with the footprints exactly within the FOV of NORSTAR GILL MSP.

\subsection{Magnetic Field Models}

[7] Figure 1a represents the modeled magnetic field configuration at 08:38:49 UT based upon the AM-03 version of Kubyshkina et al.'s [2011] adjusted magnetic field model. The AM-03 model is by far the most advanced version of Kubyshkina et al.'s event-adaptive magnetospheric model series. Under the assumption of global pressure balance, in situ measurements of plasma thermal pressure are involved in the AM- 03 model to adjust total current intensity as well as additional current sheet tilt. In this regard, the AM-03 model is supposed to be the most accurate version in modeling the magnetic field configuration in the vicinity of the 
(a)

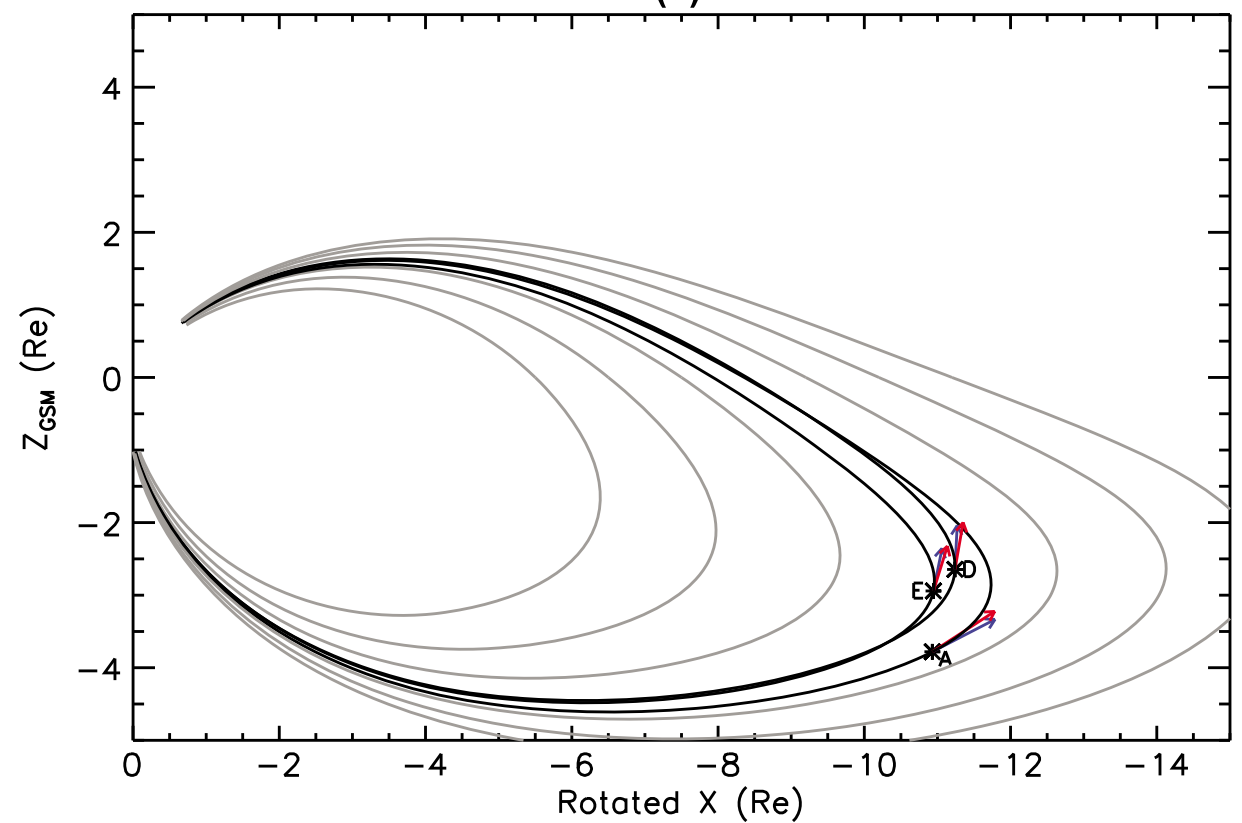

(b)

THD - 2009/02/05 08:38:49 UT

(c)
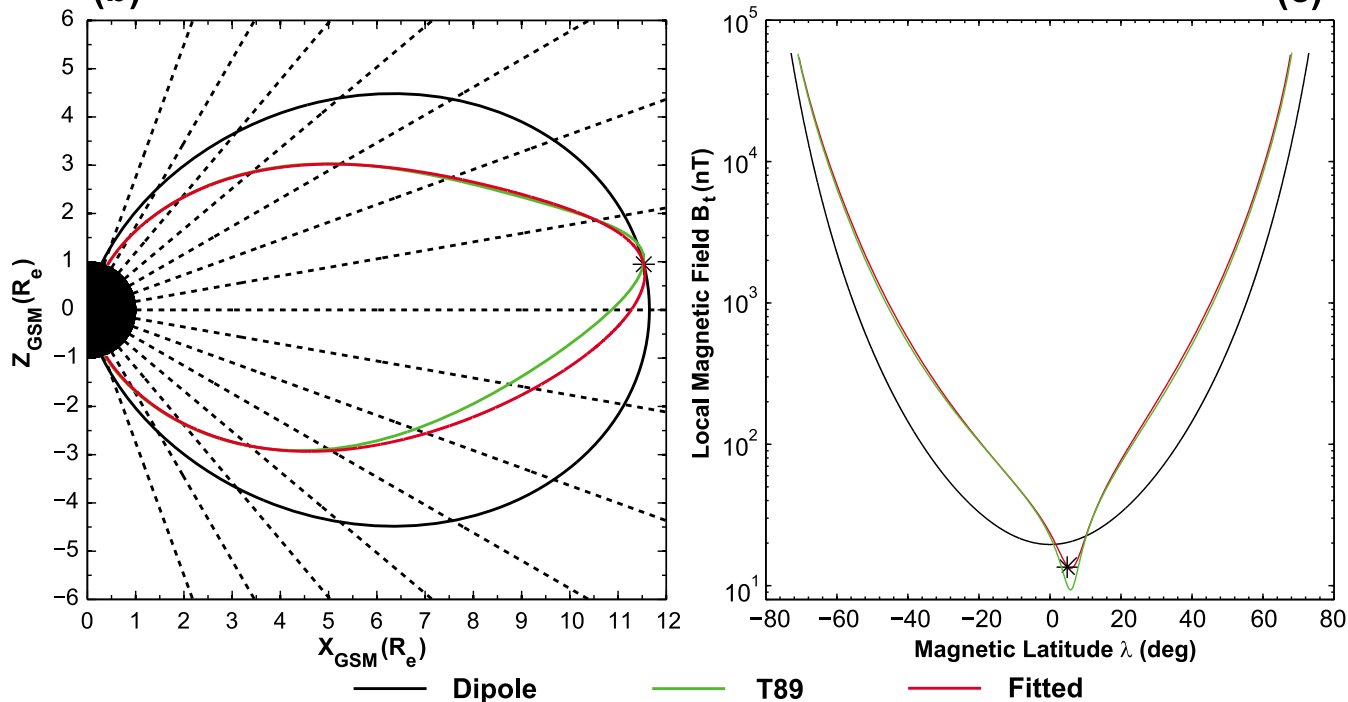

Figure 1. (a) The Kubyshkina et al. [2011] adaptive magnetic field configuration at 08:38:49 UT on February 5,2009 . The projected plane is rotated $30^{\circ}$ on the GSM X-Y plane to account for the average MLT sector of three inner THEMIS probes (THA, THD, and THE), which are shown as black asterisks. Blue and red arrows denote respectively the modeled and observed magnetic field vector for each probe. (b) Modeled magnetic field configuration and (c) magnetic field amplitude along the field line that crossed the THD location in the GSM XZ plane, color coded for three specified magnetic field models.

neutral sheet. Incorporation of global pressure balance makes the model more applicable in quasi-stable magnetospheric condition, which was satisfied for the interval of our interest. The projected plane is rotated $30^{\circ}$ on the GSM X-Y plane to account for the average MLT sector of three probes (THA, THD, and THE). The three probes, shown as black asterisks, were closely spaced. THD and THE were close to the neutral sheet, while THA was somewhat off-equator. Blue and red arrows denote respectively the modeled and actually observed magnetic field vector for the three probes, which indicates good agreement within the expected error bounds. Figures $1 \mathrm{~b}$ and $1 \mathrm{c}$ show the model results of magnetic field configuration in the GSM XZ plane and the magnetic field amplitude along the field line that crossed the THD location, color coded for different magnetic field models. Compared to the dipole field (black), the field line is distinctly distorted and exhibits substantial stretching for both the Tsyganenko 89 (T89, green) model [Tsyganenko, 1989] and the AM-03 model (red). The two non-dipolar magnetic fields are weaker than the dipole field in the equatorial region but become much stronger at higher latitudes. In addition, both T89 and AM-03 demonstrate a tilted 
magnetic field configuration at $\mathrm{L}=11.5$ with the presence of minimum $\mathrm{B}$-field $\left(\mathrm{B}_{\min }\right)$ at $\lambda=4.7^{\circ}$. For the magnetic latitude interval within a few degrees of $\mathrm{B}_{\text {min }}$ location where intense $\mathrm{ECH}$ emissions are characteristically observed, there is about a factor of 2 decrease in magnetic field strength in contrast to the dipole field results. Comparison between the T89 and AM-03 model results indicates noticeable differences especially for $Z_{\mathrm{GSM}}<\sim 1 \mathrm{R}_{\mathrm{e}}$. We also note that the modeled non-dipolar magnetic longitudes are not constant for the entire field line (not shown), indicative of a threedimensional magnetic field geometry. However, the displacement is rather minor $\left(<\sim 5^{\circ}\right)$ and the difference between the amplitude of the magnetic field along the 3-D field line and the amplitude along the projection of the field line on the GSM XZ plane is very small $(<\sim 2 \%)$ for a broad latitude range of $|\lambda| \leq 65^{\circ}$.

[8] The distinct deviation of realistic ambient magnetic field from the dipolar geometry for the event of interest can introduce considerable changes in resonant wave-particle interaction processes and thus affect the scattering loss of plasma sheet electrons for diffuse auroral precipitation, which will be investigated in detail in sections 3 and 4 .

\subsection{ECH Wave Model}

[9] To obtain the detailed information of ECH emissions, we look into an 8-s interval of 08:38:43-08:38:51 UT when THD was run in a wave burst mode and the raw data samples $(8192 / \mathrm{sec})$ from all three sensors were saved. THD EFI wave burst mode measurements on the two spin-plane sensors were despun according to the sunpulse spin-phase information and converted into the Despun Sun L-vectorZ (DSL) coordinates. Here the DSL-z axis is along the spin axis, DSL-y is defined as the cross-product of DSL-z and the Sun direction, and DSL-x completes the right-hand coordinate system, pointing approximately toward the Sun. The DSL coordinates are fairly close to the standard GSE coordinates under the standard spin-axis orientation for the inner THEMIS probes, including THD. The three components of the raw electric field were subsequently band passed for frequencies $f>f_{\text {ce }}$ (where $f_{\text {ce }}$ is electron gyrofrequency), the temporal variations of which are shown in Figures $2 \mathrm{a}-2 \mathrm{c}$ for the band-passed electric field in DSL_x, DSL_y, and DSL_z direction, respectively. It is clearly captured that the $\mathrm{ECH}$ wave activity was very intense with instantaneous peak wave amplitude occasionally above $\sim 5 \mathrm{mV} / \mathrm{m}$ and that the axial (DSL_z) electric field was smaller than those in the spin plane. The wave structures were very spiky and frequently showed repetitive bursts of wave intensity with periods less than $1 \mathrm{~s}$, very similar to those reported by Liang et al. [2010]. To determine the average wave propagation direction we have performed a maximum variance analysis (MVA) on the above ECH band-passed electric fields. Readers are referred to Liang et al. [2010] for more details about the MVA method and its calculation procedure. The eigen-vector corresponding to the largest eigen-value, which is identical to the wave vector direction for an electrostatic wave, was found to be $(0.55,-0.81,0.21)$ in the DSL coordinates. Referring to the concurrent FGM measurements, we infer that the waves have a very large wave normal angle with respect to the ambient magnetic field. The average spectral intensity of the wave electric field along the MVA vector is presented in Figure 2d. Compared to the ECH frequency bands $\left(f / f_{\text {ce }}>1.0\right)$, the spectral intensities of chorus emissions at $f<f_{\text {ce }}$ are much weaker by a few orders of magnitude and very close to the background noise level. To apply the least squares Gaussian fits to the first two ECH harmonic bands, we have assumed that the ECH waves have a Gaussian frequency distribution given by

$$
I_{E}(f)=A \exp \left[-\left(\frac{f-f_{m}}{\Delta f}\right)^{2}\right],\left(f_{l c}<f<f_{u c}\right)
$$

where $I_{E}$ is the power spectral intensity of wave electric field (in $\left.(\mathrm{mV} / \mathrm{m})^{2} / \mathrm{Hz}\right), f_{m}$ and $\Delta f$ are the frequency of maximum wave power and bandwidth, respectively. $f_{l c}$ and $f_{u c}$ are the lower and upper cutoffs to the wave spectrum outside which the wave power is assumed to be zero, and $A$ is a normalization factor given by

$$
A=\frac{E_{w}^{2}}{\Delta f} \frac{1}{\pi^{3 / 2}}\left[\operatorname{erf}\left(\frac{f_{m}-f_{l c}}{\Delta f}\right)+\operatorname{erf}\left(\frac{f_{u c}-f_{m}}{\Delta f}\right)\right]^{-1},
$$

where $E_{w}$ is the wave electric field amplitude in units of $\mathrm{mV} /$ $\mathrm{m}$ and erf is the error function. After removing the estimated noise threshold value at $1.5 \times 10^{-6}\left(\mathrm{mV}^{2} / \mathrm{m}^{2} / \mathrm{Hz}\right)$, we have fitted reasonably the $\mathrm{ECH}$ wave power frequency distribution by two Gaussian functions, that is, for the first harmonic band $\left(f_{l c} / f_{c e}=1.1\right.$ and $\left.f_{u c} / f_{c e}=1.9\right), E_{w}=1.25 \mathrm{mV} / \mathrm{m}, f_{m} / f_{c e}=1.38$, and $\Delta f l f_{c e}=0.07$; for the second harmonic band $\left(f_{l c} / f_{c e}=2.1\right.$ and $\left.f_{u c} / f_{c e}=2.9\right), E_{w}=0.07 \mathrm{mV} / \mathrm{m}, f_{m} / f_{c e}=2.76$, and $\Delta f / f_{c e}=$ 0.1. Clearly, the first ECH harmonic band contains the majority of the power of electrostatic emissions and therefore plays a dominant role in the interactions with plasma sheet electrons.

[10] To evaluate the scattering effect of ECH emissions also requires information on the wave propagation characteristics including wave normal angle and wave power spectrum along the particle bounce trajectory, which however cannot be readily obtained from the measurements. In this study we use the ray tracing HOTRAY code, which has been designed to trace any type of electrostatic or electromagnetic wave mode in a hot, magnetized, linearly unstable plasma containing several electron and ion species with Maxwellian type components at different temperatures. Readers are referred to a number of earlier studies [e.g., Horne, 1989; Horne and Thorne, 1993, 1994; Horne et al., 2003] for more details of the HOTRAY methodology.

[11] To perform the HOTRAY ray tracing simulations, we have selected the electron phase space density (PSD) data of the ESA instrument onboard THD at 08:38:13 UT when the probe was very close to the $\mathrm{B}_{\min }$ location. With the available PSDs as a function of pitch angle $\left(\alpha_{0}\right)$ for 29 energy channels from $12 \mathrm{eV}$ to $26.4 \mathrm{keV}$, as shown in Figure $3 \mathrm{a}$, we have fitted the observed electron distribution by a sum of subtracted Maxwellian components given by [e.g., AshourAbdalla and Kennel, 1978]

$$
\begin{aligned}
f_{i}\left(v_{\perp}, v_{\|}\right)= & \frac{n_{i}}{\pi^{3 / 2} \alpha_{\perp i}^{2} \alpha_{\| i}} \exp \left(-\frac{v_{\|}^{2}}{\alpha_{\| i}^{2}}\right)\left[\Delta_{i} \exp \left(-\frac{v_{\perp}^{2}}{\alpha_{\perp i}^{2}}\right)\right. \\
& \left.+\frac{1-\Delta_{i}}{1-\beta_{i}}\left(\exp \left(-\frac{v_{\perp}^{2}}{\alpha_{\perp i}^{2}}\right)-\exp \left(-\frac{v_{\perp}^{2}}{\beta_{i} \alpha_{\perp i}^{2}}\right)\right)\right]
\end{aligned}
$$


THD

(2009/02/05 08:38:43 - 08:38:51 UT)
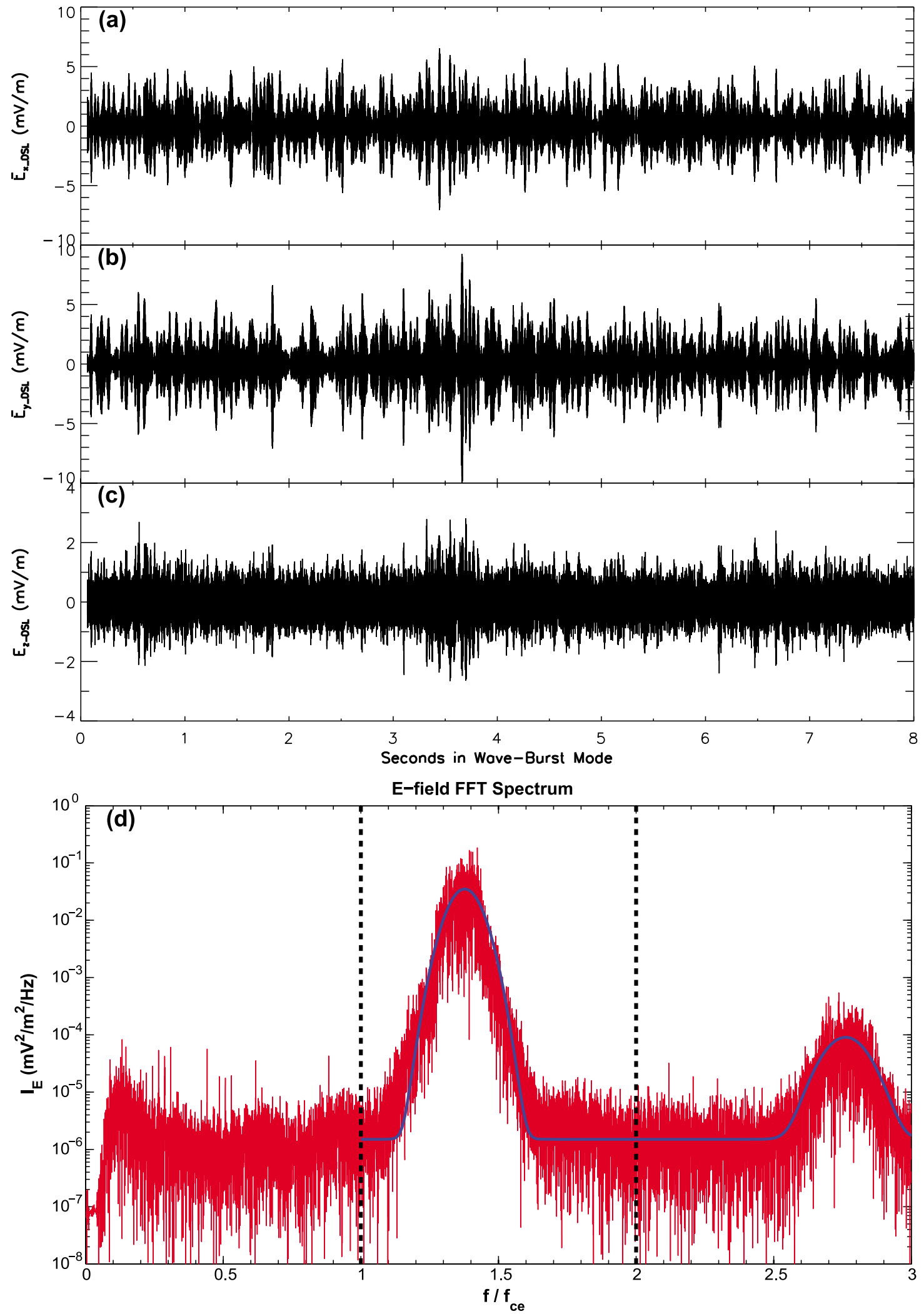

Figure 2. ECH band-passed electric field in the DSL coordinates during the wave-burst mode interval of 08:38:43-08:38:51 UT in (a) DSL_x, (b) DSL_y, and (c) DSL_z direction. (d) Average spectral intensity of the wave electric field along the MVA vector with the superimposed Gaussian fits to the first two ECH harmonic bands (blue curves). 
(a)

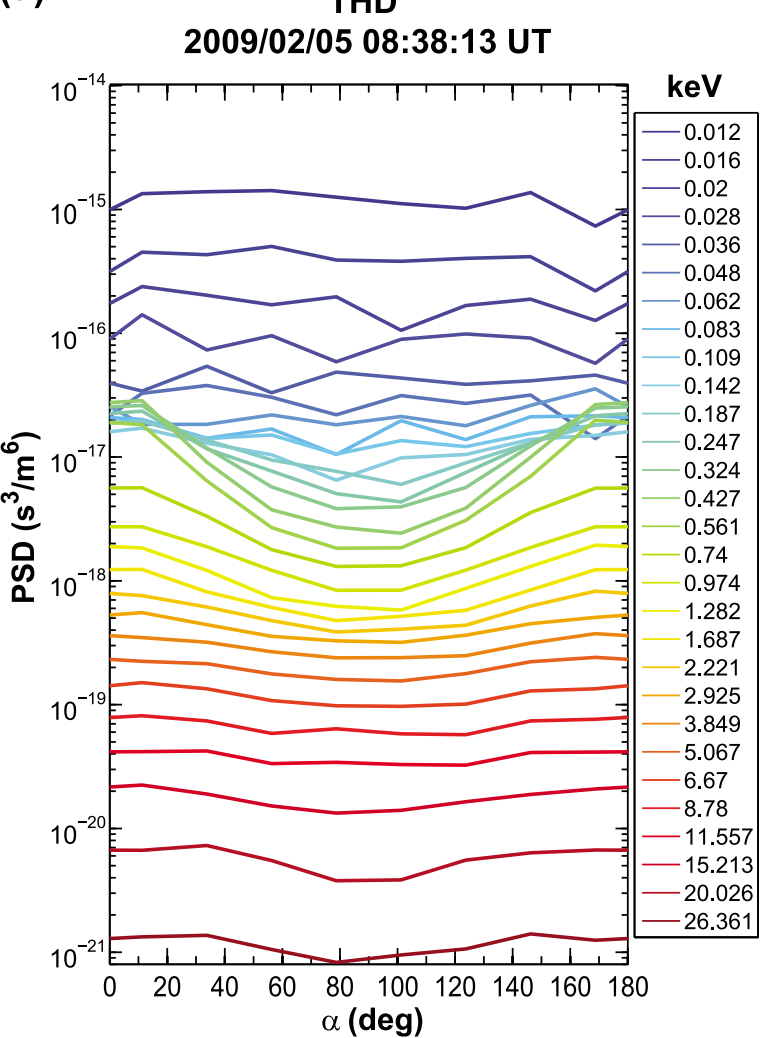

(b)
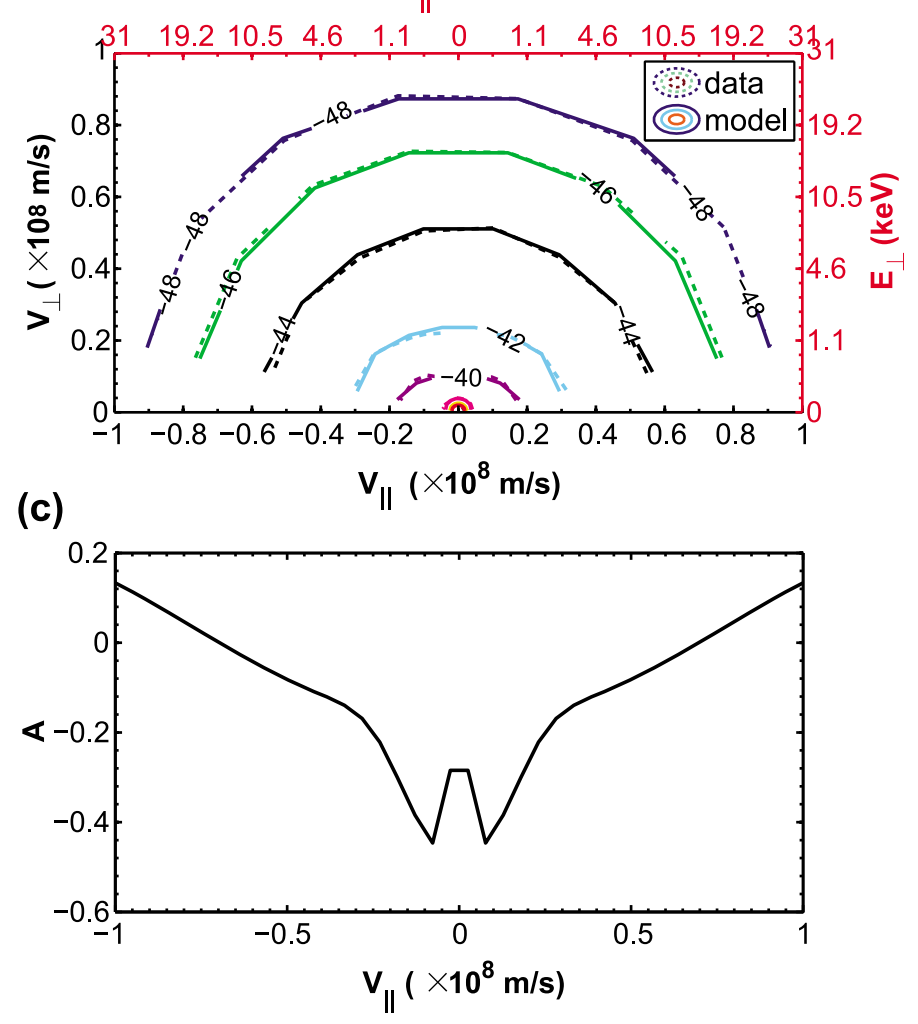

Figure 3. (a) Electron phase space density (PSD) in $\mathrm{s}^{3} / \mathrm{m}^{6}$ as a function of pitch angle for various energy levels $(12 \mathrm{eV}-26.4 \mathrm{keV})$ observed from the THD ESA instrument at 08:38:13 UT. (b) Contours of the electron PSD in $\ln \left(\mathrm{s}^{3} / \mathrm{m}^{6}\right)$ as a function of perpendicular and parallel electron velocity $(\mathrm{m} / \mathrm{s})$ (or perpendicular and parallel kinetic energy ( $\mathrm{keV}$ ) indicated by the upper red axis). The dotted lines show the ESA electron PSD data and solid lines represent the contours of electrons PSD computed from the analytical model. (c) The electron anisotropy as a function of parallel electron velocity $(\mathrm{m} / \mathrm{s})$.

where the subscript " $i$ " denotes the $i$ th component of electrons, $f_{i}$ is the phase space density, $n_{i}$ is the electron density, $\alpha_{\perp i}$ and $\alpha_{\| i}$ are the thermal velocities perpendicular and parallel to the ambient magnetic field, and $\Delta i$ and $\beta_{i}$ determine the width and the depth of the loss cone, respectively. The total number of components and all the parameters are given in Table 1. While THEMIS ESA pitch angle resolution is not high enough to provide the details of PSD at smaller pitch angles, the parameters $\Delta i$ and $\beta_{i}$ are adjusted to provide a loss cone $<1^{\circ}$, corresponding to that calculated from the AM-03 magnetic field model. Contours of the model distribution are shown in Figure $3 \mathrm{~b}$ by the dashed lines. The contours fit the measured contours reasonably well. Figure $3 \mathrm{c}$ represents the effective pitch angle anisotropy (A) for the resonant electrons [e.g., Kennel and Petschek, 1966; Li et al., $2009 \mathrm{~b}$ ] as a function of parallel electron velocity $v_{\|}$.

[12] On the basis of the modeled hot electron distributions and the measured magnetic field strength and electron density at the $\mathrm{B}_{\min }$ location $\left(\lambda=4.7^{\circ}\right)$, we have adopted the HOTRAY hot plasma dispersion relation solver to compute the wave dispersion and local growth rate for six specific frequencies in the first $\mathrm{ECH}$ harmonic band (1.2 $f_{\mathrm{ce}}$ to $1.7 f_{\mathrm{ce}}$ with an increment of $0.1 f_{\text {ce }}$ ) by varying wave normal angle $\theta$. While the wave number $\mathrm{k}$ remains almost constant with wave normal angle, the temporal growth rate shows a strong dependence on wave normal angle, consistent with the previous studies [e.g., Horne and Thorne, 2000; Horne et al., 2003; Ni et al., 2011a]. For instance, the peak wave normal angle $\theta_{0}$ that corresponds to the maximum growth rate is found to be $89.1^{\circ}$ for $1.2 f_{\mathrm{ce}}, 89^{\circ}$ for $1.3 f_{\mathrm{ce}}, 89.6^{\circ}$ for $1.4 f_{\mathrm{ce}}$, and $89.7^{\circ}$ for $1.5 f_{\text {ce }}$, showing that ECH waves can only grow at high angles close to $90^{\circ}$ with respect to the ambient magnetic field. Since strong ECH emissions are characteristically confined within a few degrees of the source region [e.g., Gough et al., 1979; Belmont et al., 1983; Roeder and Koons, 1989; Meredith et al., 2009; Ni et al., 2011c], we assume that the dominant ECH wave activity is present between $4.7^{\circ} \pm 2^{\circ}$ during the event of interest. This enables

Table 1. Hot Electron Components Used to Model the THD ESA Electron Phase Space Density Distribution at 08:38:11 UT on February 5, 2009 by a Sum of Subtracted Maxwellian Functions

\begin{tabular}{cccccc}
\hline Component & $T_{\perp}(\mathrm{eV})$ & $T_{\|}(\mathrm{eV})$ & $\mathrm{N}_{\mathrm{e}}\left(\mathrm{m}^{-3}\right)$ & $\Delta$ & $\beta$ \\
\hline 1 & 2.0 & 2.3 & $8.0 \times 10^{5}$ & 1.0 & 0.5 \\
2 & 10.2 & 106.4 & $3.1 \times 10^{4}$ & 0.8 & 0.1 \\
3 & 160.1 & 569.6 & $1.0 \times 10^{5}$ & 0.01 & 0.01 \\
4 & 1422.1 & 3441.0 & $1.2 \times 10^{5}$ & 0.3 & 0.1 \\
5 & 3044.5 & 4432.1 & $9.2 \times 10^{4}$ & 0.1 & 0.9 \\
\hline
\end{tabular}


us to apply a linear interpolation of peak wave normal angle $\theta_{0}$ for the specified magnetic latitude interval of wave presence by assuming that $\theta_{0}=90^{\circ}$ at the two latitude boundaries to construct the latitudinal variation of $\theta_{0}$ during propagation for each considered wave frequency. While we have the least information for the angular width of $\mathrm{ECH}$ wave spectrum $(\delta \theta)$, we have approximately taken $\delta \theta=90^{\circ}-\theta_{0}$. To obtain the parallel wave number $k_{0, \|}\left(=k_{0, \perp} / \tan \theta_{0}\right)$, the HOTRAY hot plasma dispersion relation solver computes the wave number at the $\mathrm{B}_{\min }$ location and we assume the perpendicular wave number $\left(k_{0, \perp}\right)$ unchanged with wave normal angle. $\delta k_{\|}$is determined by $\delta k_{\|}=\tan \left(\theta_{0}-\delta \theta\right)-k_{0, \|}$. Readers are referred to $\mathrm{Ni}$ et al. [2011a] for more details of how to determine these wave parameters. The latitudinal variations of ECH wave power distribution, modeled based upon the HOTRAY simulations, provide necessary inputs for quantifying bounce-averaged diffusion rates by broadband $\mathrm{ECH}$ emissions.

\section{Evaluation of Bounce-Averaged Diffusion Coefficients by ECH Emissions}

\subsection{Equations of Diffusion Coefficient Computation}

[13] Under the assumption that the ECH wave electric field spectrum has the form of

$$
\begin{aligned}
\left|E_{k}\right|^{2}= & C k_{\perp}^{2} \exp \left[-\left(\frac{k_{\perp}}{k_{0, \perp}}\right)^{2}\right] \cdot\left\{\exp \left[-\left(\frac{k_{\|}-k_{0, \|}}{\delta k_{\|}}\right)^{2}\right]\right. \\
& \left.+\exp \left[-\left(\frac{k_{\|}+k_{0, \|}}{\delta k_{\|}}\right)^{2}\right]\right\}
\end{aligned}
$$

with a normalization constant

$$
C=\frac{4 \pi^{3 / 2}}{k_{0, \perp}^{4} \delta k_{\|}} V\left|E_{w}\right|^{2}
$$

and that the parallel group velocity is small compared to the electron parallel velocity (i.e., $\partial \omega_{k} / \partial k_{\|} \ll v_{\|}$), the local pitch angle diffusion coefficient for electrons due to electrostatic $\mathrm{ECH}$ waves (in units of $\mathrm{s}^{-1}$ ) at any specified wave frequency can be given by [e.g., Horne and Thorne, 2000; Ni et al., 2011a]

$$
\begin{aligned}
D_{\alpha \alpha}= & \frac{\sqrt{\pi}}{2}\left(\frac{e}{m_{e}}\right)^{2} \frac{E_{w}{ }^{2}}{k_{0, \perp}^{2} \delta k_{\|}} \frac{\exp (-\mu)}{v^{5} \cos \alpha} \sum_{N=-\infty}^{+\infty}\left(\frac{N \Omega_{e} / \gamma-\omega_{k} \sin ^{2} \alpha}{\sin \alpha \cos \alpha}\right)^{2} \\
& \cdot I_{N}(\mu)\left\{\exp \left[-\left(\zeta_{N}^{-}\right)^{2}\right]+\exp \left[-\left(\zeta_{N}^{+}\right)^{2}\right]\right\}
\end{aligned}
$$

where $k_{\perp}$ and $k_{\|}$are the components of the wave vector perpendicular and parallel to the ambient magnetic field $\mathbf{B}_{0}$, respectively, $e / m_{e}$ is the electron charge to mass ratio, $N$ is the order of resonance harmonics, $\Omega_{e}=\left|e B_{0} / m_{e}\right|$ is the angular electron gyrofrequency, $\omega_{k}$ is the wave frequency, $\gamma=\left(1-v^{2} / c^{2}\right)^{-1 / 2}$ is the Lorentz factor with $v$ as the electron velocity and $c$ the speed of light, $\alpha$ is the electron pitch angle, $V$ is the plasma volume, $\zeta_{N}^{ \pm}=\frac{\omega_{k}-N \Omega_{e} / \gamma}{\delta k_{\|} v \cos \alpha} \pm \frac{k_{0, \|}}{\delta k_{\|}}$, and $I_{N}(\mu)$ is the modified Bessel function with the argument $\mu=$ $k_{0, \perp}^{2} v_{\perp}^{2} /\left(2 \Omega_{e}^{2}\right)$. Local cross diffusion rate $D_{\alpha p}$ and momentum diffusion rate $D_{p p}$ can be obtained by [e.g., Lyons, 1974; Glauert and Horne, 2005; Albert, 2007]

$$
\begin{aligned}
& D_{\alpha p}=D_{\alpha \alpha}\left[\frac{\sin \alpha \cos \alpha}{N \Omega_{e} /\left(\gamma \omega_{k}\right)-\sin ^{2} \alpha}\right], \\
& D_{p p}=D_{\alpha \alpha}\left[\frac{\sin \alpha \cos \alpha}{N \Omega_{e} /\left(\gamma \omega_{k}\right)-\sin ^{2} \alpha}\right]^{2} .
\end{aligned}
$$

Following Orlova and Shprits [2010] and Ni et al. [2011d], for non-dipolar fields, if the field line lies in a plane perpendicular to the magnetic equator plane, the quasi-linear bounce-averaged diffusion coefficients along the particle bounce trajectory are written as

$$
\left\langle D_{\alpha \alpha}\right\rangle=\frac{\int_{\lambda_{m, s}}^{\lambda_{m, n}} \frac{D_{\alpha \alpha}(\alpha)}{\cos \alpha}\left(\frac{\tan \alpha_{e q}}{\tan \alpha}\right)^{2} \sqrt{r^{2}+\left(\frac{\partial r}{\partial \lambda}\right)^{2}} d \lambda}{\int_{\lambda_{m, s}}^{\lambda_{m, n}} \sec \alpha \sqrt{r^{2}+\left(\frac{\partial r}{\partial \lambda}\right)^{2}} d \lambda},
$$

$$
\begin{gathered}
\left\langle D_{\alpha p}\right\rangle=\frac{\int_{\lambda_{m, s}}^{\lambda_{m, n}} \frac{D_{\alpha p}(\alpha)}{\cos \alpha} \frac{\tan \alpha_{e q}}{\tan \alpha} \sqrt{r^{2}+\left(\frac{\partial r}{\partial \lambda}\right)^{2}} d \lambda}{\int_{\lambda_{m, s}}^{\lambda_{m, n}} \sec \alpha \sqrt{r^{2}+\left(\frac{\partial r}{\partial \lambda}\right)^{2}} d \lambda}, \\
\left\langle D_{p p}\right\rangle=\frac{\int_{\lambda_{m, s}}^{\lambda_{m, n}} \frac{D_{p p}(\alpha)}{\cos \alpha} \sqrt{r^{2}+\left(\frac{\partial r}{\partial \lambda}\right)^{2}} d \lambda}{\int_{\lambda_{m, s}}^{\lambda_{m, n}} \sec \alpha \sqrt{r^{2}+\left(\frac{\partial r}{\partial \lambda}\right)^{2}} d \lambda},
\end{gathered}
$$

where $r$ is radial distance to the Earth's center, $\lambda$ is magnetic latitude, and $\lambda_{m, s}$ and $\lambda_{m, n}$ are the mirror latitude of particles on the southern and northern hemisphere, respectively, which are strictly dependent on the field line configuration and the field strength of adopted magnetic field model.

[14] Theoretically, quantification of diffusion rates requires integration over the entire $\mathrm{ECH}$ frequency band. Here we follow the weighting method developed by $\mathrm{Ni}$ et al. [2011a] by calculating the diffusion rates at a number of representative frequencies and introducing reasonable weighting factors at each wave frequency to obtain the overall diffusion coefficients efficiently and reasonably. That is, the overall bounce-averaged diffusion coefficients are computed from

$$
\langle D\rangle_{\text {overall }}=\sum_{j=1}^{M} R_{j}\langle D\rangle_{j}
$$

with the weighting factor for the $j$ th wave frequency given by

$$
R_{j}=\frac{\left(I_{E}\right)_{j}}{\sum_{j=1}^{M}\left(I_{E}\right)_{j}} .
$$


(a)

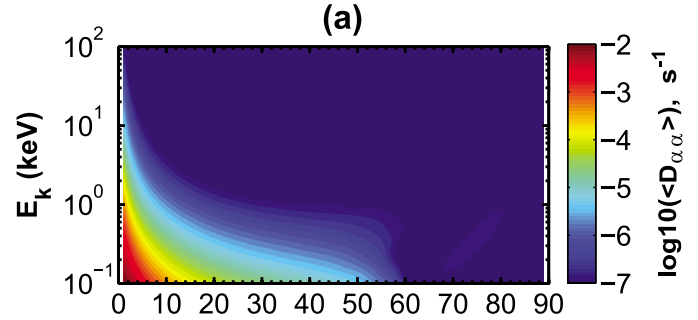

(b)

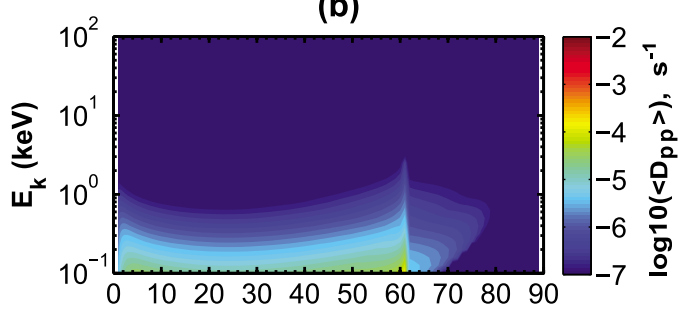

(c)

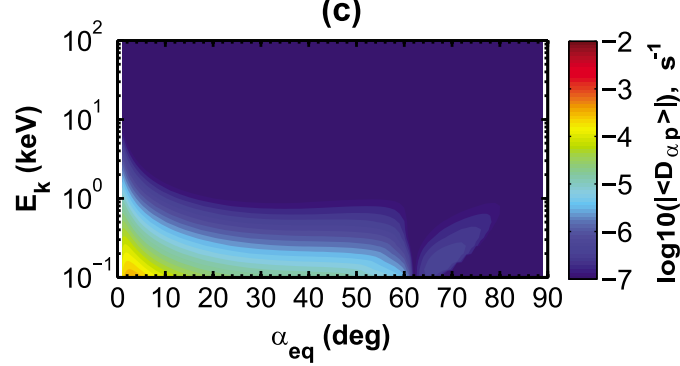

Figure 4. $(\mathrm{a}-\mathrm{c})$ Net (overall) bounce-averaged resonant diffusion rates $\left\langle D_{\alpha \alpha}\right\rangle\left\langle D_{p p}\right\rangle$, and $\left|\left\langle D_{\alpha p}\right\rangle\right|$ due to the observed first ECH harmonic band as a function of equatorial pitch angle and electron kinetic energy at $\mathrm{L}=11.5$, calculated using the Kubyshkina et al. [2011] adaptive magnetic field model.

Here $M$ is the number of frequency considered for $\mathrm{ECH}$ frequency band.

\subsection{Resonant Scattering Rates by ECH Waves}

[15] Using the event-adaptive magnetic field model and the ECH wave model developed in section 2 for the event under consideration, we can then incorporate modeled parameters into the diffusion rate equations described in section 3 to compute bounce-averaged scattering rates. Our calculations include contributions from the $\mathrm{N}=-10$ to $\mathrm{N}=$ 10 cyclotron harmonic resonances and the Landau resonance $\mathrm{N}=0$. Because most electrostatic wave power is within 1$2 f_{\text {ce }}$, we focus on the first ECH harmonic band at six specific frequencies $\left(1.2 f_{\mathrm{ce}}\right.$ to $1.7 f_{\mathrm{ce}}$ with an increment of $\left.0.1 f_{\mathrm{ce}}\right)$. We also assume that the ECH wave spectral intensities and electron density are constant with latitude.

[16] The overall (net) bounce-averaged pitch angle diffusion coefficients $\left\langle D_{\alpha \alpha}\right\rangle$, momentum diffusion coefficients $\left\langle D_{p p}\right\rangle$, and (pitch angle, momentum) mixed diffusion coefficients $\left\langle D_{\alpha p}\right\rangle$ due to the first ECH harmonic band are shown in Figure 4 as a function of equatorial pitch angle $\alpha_{e q}$ and kinetic energy $E_{k}$. Clearly, there is a strong dependence of ECH wave driven resonant scattering rates on electron energy and equatorial pitch angle. For the event of interest, most intense scattering losses by ECH waves occur for plasma sheet electrons in the energy range from $100 \mathrm{eV}$ to $\sim 5 \mathrm{keV}$, at a rate of $>10^{-4} \mathrm{~s}^{-1}$ that increases with decreasing energy. In contrast, the scattering effect of ECH waves to electrons $>\sim 10 \mathrm{keV}$ is very inefficient, at a rate of the order of $10^{-6} \mathrm{~s}^{-1}$ or less. In addition, intense ECH wave scattering is commonly confined to $\alpha_{e q}<30^{\circ}$, which is consistent with the previous results in the inner magnetosphere [e.g., Horne and Thorne, 2000; Thorne et al., 2010; $\mathrm{Ni}$ et al., 2011a]. As electron energy increases (up to $\sim 5 \mathrm{keV}$ ), a smaller population of plasma sheet electrons within a narrower pitch angle interval around the loss cone undergoes efficient pitch angle scattering with $\left\langle D_{\alpha \alpha}\right\rangle>$ $10^{-5} \mathrm{~s}^{-1}$. Compared to pitch angle diffusion rates, ECH wave induced momentum diffusion rates and mixed diffusion rates are generally relatively small, suggesting that $\mathrm{ECH}$ emissions play a minor role in energizing plasma sheet electrons. The peak in $\left\langle D_{p p}\right\rangle$ at $E_{k} \sim 100 \mathrm{eV}$ and $\alpha_{e q} \sim 60^{\circ}$ mainly results from the $\mathrm{N}=1$ resonant diffusion by $\mathrm{ECH}$ emissions at $1.3 f_{\text {ce }}$ for which $k_{\|}$values are largest.

[17] A more detailed examination of pitch angle scattering of plasma sheet electrons by ECH waves for the considered event at $\mathrm{L}=11.5$ is shown in Figure 5a for six specific energies ranging from $200 \mathrm{eV}$ to $10 \mathrm{keV}$. Evidently, the observed ECH emissions can result in efficient precipitation loss of plasma sheet electrons below $\sim 5 \mathrm{keV}$. A good estimate for the electron loss timescale is given by $1 /\left\langle D_{\alpha \alpha}\right\rangle$ where the bounce-averaged electron pitch angle scattering rate $\left\langle D_{\alpha \alpha}\right\rangle$ is evaluated at the equatorial loss cone angle $\alpha_{L C}$

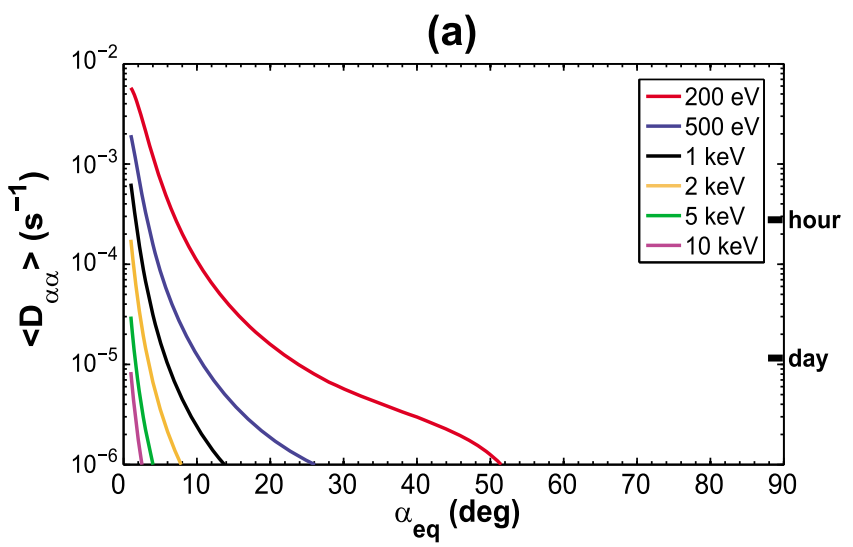

(b)

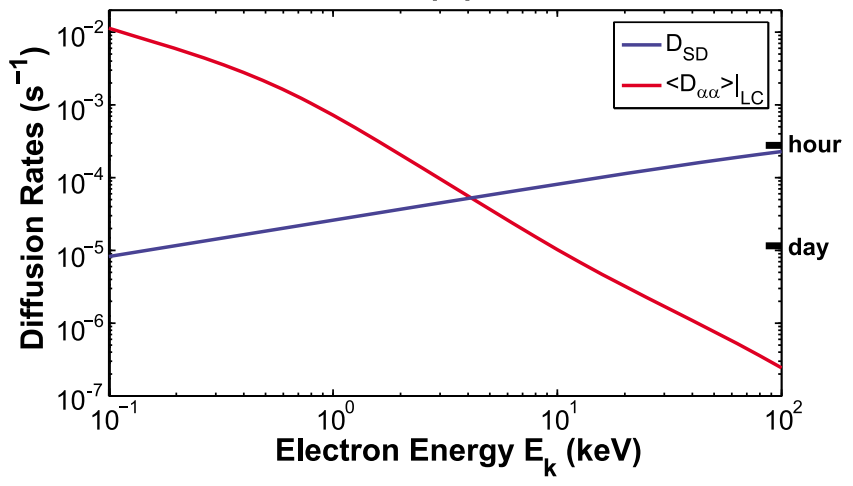

Figure 5. (a) ECH wave-induced bounce-averaged pitch angle diffusion coefficients as a function of equatorial pitch angle at $\mathrm{L}=11.5$ for six specified energies from $200 \mathrm{eV}$ to $10 \mathrm{keV}$. (b) Comparison of $\left\langle D_{\alpha \alpha}\right\rangle$ near the loss cone angle with strong diffusion rate $D_{S D}$. 
[e.g., Shprits et al., 2006; Summers et al., 2007a, 2007b; Summers and Ni, 2008; Albert and Shprits, 2009]. In light of the realistic, non-dipolar magnetic field shown in Figure 1, we find $\alpha_{L C}=0.88^{\circ}$. The estimated lifetime is much less than one hour for $200 \mathrm{eV}-1 \mathrm{keV}$ electrons, about two hours for $2 \mathrm{keV}$ electrons, a third of a day or so for $5 \mathrm{keV}$ electrons, and above one day for $10 \mathrm{keV}$. The loss timescales for $200 \mathrm{eV}-\sim 5 \mathrm{keV}$ electrons are shorter than their transport periods for drift between midnight and dawn that generally take several hours. Consequently, these plasma sheet electrons can be significantly depleted during the transport process. To investigate the efficiency of ECH waves in driving the precipitation loss of plasma sheet electrons, we also compare the pitch angle scattering rates at the edge of loss cone $\left.\left\langle D_{\alpha \alpha}\right\rangle\right|_{L C}$ with the strong diffusion rate $D_{S D}$ determined by [Kennel, 1969]

$$
D_{S D}=\frac{2\left(\alpha_{L C}\right)^{2}}{\tau_{B}}
$$

where $\tau_{B}$ is the electron bounce period along the entire field line determined by

$$
\tau_{B}=\frac{2}{v} \int_{\lambda_{m, s}}^{\lambda_{m, n}} \sec \alpha \sqrt{r^{2}+\left(\frac{\partial r}{\partial \lambda}\right)^{2}} d \lambda
$$

with $v$ as the electron thermal speed. The results, shown in Figure $5 \mathrm{~b}$, indicate that as the electron energy increases from $100 \mathrm{eV}$ to $100 \mathrm{keV}$, the timescale of strong diffusion decreases from $\sim$ one day to one hour. For electrons $>10 \mathrm{keV}$, pitch angle scattering rates near the loss cone are at least one order of magnitude smaller than the strong diffusion rates $D_{S D}$. The transition to strong diffusion with a corresponding fully or partially filled loss cone occurs below $6 \mathrm{keV}$. This suggests that $\mathrm{ECH}$ wave scattering in the outer magnetosphere is important for the lower energy portion of plasma sheet electrons leading to diffuse auroral precipitation. Since the loss cone is small and the lifetime for strong diffusion is longer at higher L-shells, it is expected that scattering depletion of plasma sheet electrons by ECH waves at higher L-shells cannot be as strong as that at lower L-shells, which can qualitatively explain the statistical occurrence of most intense diffuse auroral precipitation in the inner magnetosphere on the nightto-dawn side [Newell et al., 2009].

\section{Quantitative Analysis of Diffuse Auroral Precipitation Flux}

[18] The comparison above between pitch angle diffusion rates and strong diffusion rates indicates that only a portion of plasma sheet electrons at energies $<6 \mathrm{keV}$ suffers efficient precipitation loss at a rate approaching the strong diffusion, thereby explaining why the estimate of electron precipitation flux by LJ2011, based upon the assumption of a fully filled loss cone for all energies, produced a result larger than the actually observed value. In this section we will perform a more accurate evaluation of the precipitation flux of plasma sheet electrons at the ionospheric footprint on the basis of the ECH wave driven resonant scattering rates for the time interval of interest at $\mathrm{L}=11.5$.

[19] Figure 6a shows the GILL MSP green-line (557.7 nm) auroral observations for an hour period from 07:50 UT to
08:50 UT. For the time interval under investigation, 08:38:43-08:38:51 UT, the footprint of THD is marked as a rectangle according to the Kubyshkina et al. [2011] adaptive magnetic field mapping. Specifically, we present in Figure $6 b$ the latitudinal variation of green-line auroral intensity for the time stamp centered at 08:38:45 UT. The THD footprint obtained from the Kubyshkina et al. magnetic field was only $\sim 2^{\circ}$ in magnetic longitude west to the GILL MSP scan meridian. The gray band shows the THD footprint in magnetic latitude, say, $67.9^{\circ}-68.5^{\circ}$ with certain mapping uncertainty. It is always difficult to estimate errors in mapping, however, based on recent work using correlations between chorus modulation and pulsating aurora [Nishimura et al., 2011] and assuming that the flux tube is not near the edge of large-scale field-aligned current systems [e.g., Donovan, 1993], we assert that an uncertainty of plus/minus $0.3^{\circ}$ is reasonable. Averaging over this band gives an intensity value of $\sim 2.4 \mathrm{kR}$.

[20] In the absence of any midway auroral acceleration process, ionospheric precipitation flux $\Phi$ is purely determined by the differential energy flux of precipitation electrons inside the equatorial loss cone, which is given by [e.g., Chang, 1983]

$$
\Phi=2 \pi \frac{B_{I}}{B_{e q}} \int_{E_{1}}^{E_{2}} \int_{0}^{\alpha_{L C}} J(E, \alpha) \cos \alpha \sin \alpha d E d \alpha,
$$

where the ratio between the magnetic field strength at the ionospheric footprint and at the magnetic equator, $B_{I} / B_{e q}$, is attributed to the change of flux tube cross section when mapping from the equatorial magnetosphere to the ionosphere, $J$ is the electron differential energy flux inside the equatorial loss cone as a function of energy and pitch angle, and $E_{1}$ and $E_{2}$ are the lower and upper limit for integration over energy. Since strong diffusion driven by the $\mathrm{ECH}$ emissions does not occur for plasma sheet electrons at all energies, as described in Figure 5b, there are two subsets of plasma sheet electron population; lower energy electrons that approach strong diffusion limit with a fully filled loss cone, and higher energy electrons undergoing slower precipitation loss with a partially filled or empty loss cone. The most accurate way to obtain the electron distribution within the loss cone is to solve the modified Fokker-Planck equation [e.g., Liang et al., 2007; Thorne et al., 2010; Tao et al., 2011], but since our interest is limited to the final diffusion equilibrium, an easy approach for our evaluation purpose is to use the analytical solution under diffusion equilibrium condition as described by Kennel and Petschek [1966],

$$
J(E, \alpha)=J\left(E, \alpha_{L C}\right) \cdot \frac{I_{0}\left(Z_{0} \cdot \alpha / \alpha_{L C}\right)}{I_{0}\left(Z_{0}\right)},
$$

where $Z_{0}=\sqrt{D_{S D} /\left\langle D_{\alpha \alpha}\right\rangle \mid L C}$ is an energy-dependent parameter defining the diffusion strength near the loss cone, $I_{0}$ is the modified Bessel function of the first kind, $J\left(E, \alpha_{L C}\right)$ is the electron energy flux near the equatorial loss cone estimated from the THD ESA measurements with pitch angle information by a linear interpolation, which is shown as red curve in Figure 6c. Substituting equation (16) into equation (15) and assuming that electron energy flux is constant over the 
(a)

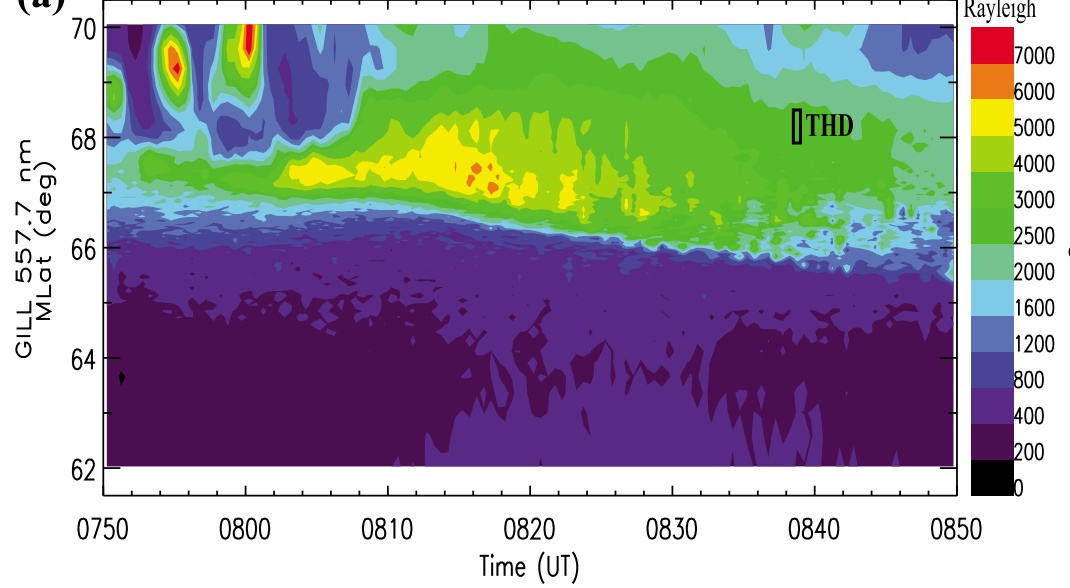

(b)

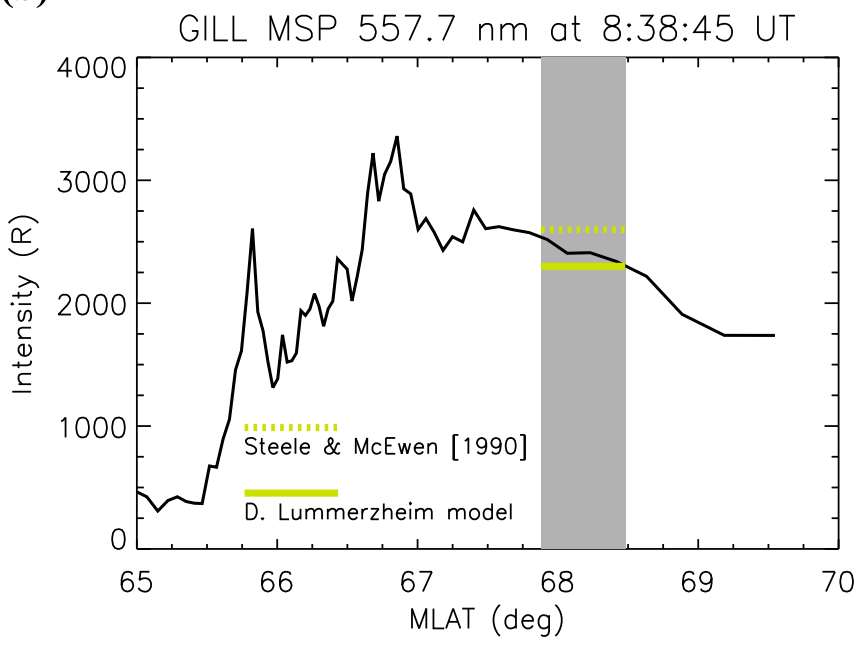

(c) Estimated THD Electron Energy Fluxes

(d)

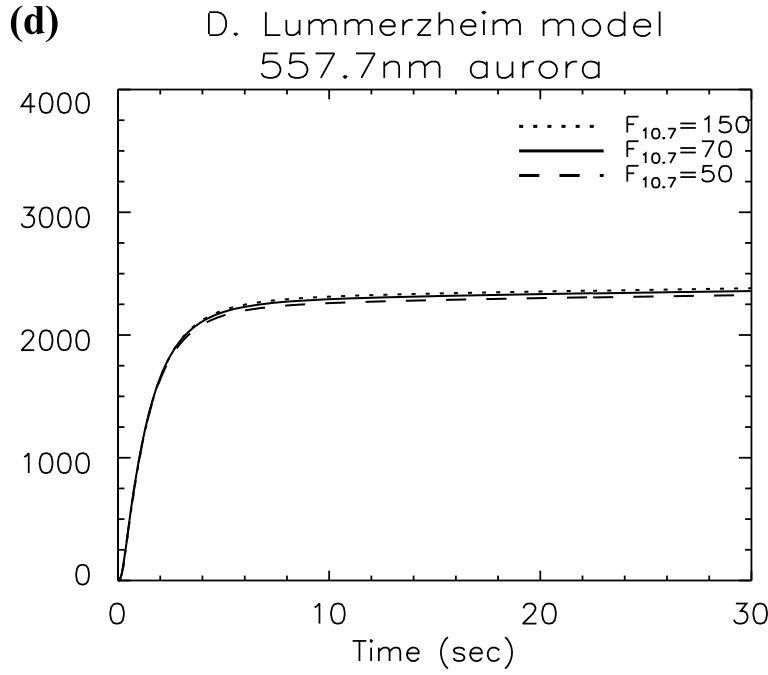

Figure 6. (a) NORSTAR GILL MSP green-line $(557.7 \mathrm{~nm})$ auroral observations from 07:50 UT to 08:50 UT on February 5, 2009. The superimposed rectangle denotes the footprint of THD for the time interval of interest, 08:38:43-08:38:51 UT. (b) The latitudinal variation of MSP green-line auroral intensity for the time stamp centered at 08:38:45 UT. The gray band shows the THD footprint in magnetic latitude, plus/minus $0.3^{\circ}$. The solid and dotted yellow horizontal lines represent respectively the modeled green-line auroral intensity using the auroral electron transport model developed by Lummerzheim [1987] and the Maxwellian-type fitting to electron precipitation flux following Steele and McEwen [1990]. (c) Electron energy flux at the equatorial loss cone (red curve) obtained from the THD ESA differential flux measurements with pitch angle information and electron precipitation flux inside the equatorial loss cone (blue curve) estimated based upon the index of loss cone filling due to ECH wave scattering. (d) Lummerzheim [1987] model results of the $557.7 \mathrm{~nm}$ auroral brightness for 3 different profiles of the neutral atmosphere with the solar F10.7 flux of 150, 70 and 50.

small pitch angle interval from $0^{\circ}$ to $\alpha_{L C}$, which is reasonable for $\alpha<\alpha_{L C} \ll 1$, after some algebra we obtain

$$
\begin{gathered}
\Phi \approx \pi \int_{E_{1}}^{E_{2}} x(E) \cdot J\left(E, \alpha_{L C}\right) d E \\
\text { with } x(E)=\frac{2 \int_{0}^{1} I_{0}\left[Z_{0}(E) \tau\right] \cdot \tau \cdot d \tau}{I_{0}\left[Z_{0}(E)\right]} .
\end{gathered}
$$

Here $x$ is the index of loss cone filling, energy dependent in association with the efficiency of ECH wave pitch angle scattering. A key and interesting conclusion from equation (17) is that the ionospheric precipitation flux keeps constant in a flux tube and is independent of the ratio of $B_{I} / B_{\text {eq }}$, in other words, the change in flux tube cross section along the particle bounce trajectory, which is physically reasonable. In our case, $x$ is equal or very close to 1 for $E_{k}<5 \mathrm{keV}$ and decreases from 0.84 to 0.24 for the ESA higher energy channels. The electron differential energy flux inside the equatorial loss cone estimated following equations (16)-(18) is shown as blue curve in Figure 6c.

[21] Using the energy spectrum of precipitating electron flux shown in Figure 6c as model input, we adopt the auroral electron transport model developed by Lummerzheim [1987] to evaluate the brightness of induced diffuse aurora. This 
model takes into account all the ionization and auroral excitation processes in the $90-200 \mathrm{~km}$ altitude range. By assuming a typical winter, midnight IRI electron profile and no auroral emission as the initial condition, we have run the model for $30 \mathrm{~s}$ (the scan period of NORSTAR MSP) during which the electron density and auroras are self-consistently calculated. A number of numerical tests have been performed, indicating that the saturation level of the $557.7 \mathrm{~nm}$ auroral brightness is independent of the initial electron density profile. Figure $6 \mathrm{~d}$ presents the model results of the $557.7 \mathrm{~nm}$ green-line auroral brightness for 3 different profiles of the neutral atmosphere, obtained using the MSISE90 model [Hedin, 1991] with the solar F10.7 flux of 150, 70 (actually observed value corresponding to the event), and 50, respectively. Insensitive to the ambient neutral atmospheric condition, the evolution of the $557.7 \mathrm{~nm}$ brightness rises from 0 (specified initial condition) and quickly saturates at $\sim 2.3 \mathrm{kR}$ (shown as solid yellow line in Figure 6b), in excellent agreement of the actual MSP observation for the $8 \mathrm{~s}$ interval of our interest. On the basis of the estimated electron precipitation flux inside the loss cone shown as blue curve in Figure 6c, LJ2011 has followed the approach of Steele and McEwen [1990] to perform a Maxwellian-type fitting to the energetic portion $(>1 \mathrm{keV})$ of the precipitating spectrum, which dominantly contribute to the $557.7 \mathrm{~nm}$ auroral emissions. Their estimate gives an auroral intensity of $\sim 2.6 \mathrm{kR}$ (shown as dotted yellow line in Figure $6 \mathrm{~b}$ ), which is reasonably consistent with the actual observation and our model result.

[22] Through two recognized methods to convert the electron precipitation fluxes to optical auroral Rayleigh values, we have obtained good agreement between the modeled and observed diffuse auroral intensities. This agreement quantitatively confirms the scenario proposed by LJ2011 that the ECH wave activity is responsible for the observed diffuse auroral precipitation in the outer magnetosphere for the event of our interest.

\section{Conclusions and Discussion}

[23] As far as we know, this study is the first attempt to quantify the scattering rates of CPS electrons by electrostatic $\mathrm{ECH}$ emissions and to simulate the subsequent ionospheric precipitation flux and resulting auroral brightness to study the magnetospheric cause of diffuse aurora in the outer CPS. We have done so through a systematic combination of quasilinear theory, realistic non-dipolar magnetic field mapping, and the concept of strong diffusion on the basis of conjugated space and ground observations.

[24] For the event of our interest, the companion paper by LJ2011 has established a causal connection between the space-borne THEMIS EFI ECH emission measurements and the ground-based NORSTAR MPI diffuse aurora observations. This study subsequently provides a detailed quantitative investigation of whether or not the observed ECH waves could be the cause of resonant scattering of plasma sheet electrons and how they contributed to the diffuse aurora at $\sim 68^{\circ}$ invariant latitude in the reported event. To fulfill the goal, realistic wave and particle observations as well as the event-adaptive AM-03 magnetic field model [Kubyshkina et al., 2011] have been adopted to perform a high fidelity numerical analysis. The substantial departure of magnetic field configuration from a dipolar geometry in the outer magnetosphere modifies dramatically resonant wave-particle interaction processes including the rate of strong diffusion. Consideration of hot electron components and use of the HOTRAY code to solve the hot plasma dispersion relation guarantees a reasonable analysis of $\mathrm{ECH}$ wave power distribution over wave frequency and wave normal angle for evaluation of resonant electron energies and bounceaveraged quasi-linear diffusion coefficients. In addition, computation of loss cone angle and bounce period in a realistic magnetic field configuration allows us to accurately differentiate between plasma sheet electrons subject to strong diffusion, quasi-strong diffusion, and weak diffusion and thus accurately model the precipitation into the highlatitude ionosphere.

[25] Our main conclusions are summarized as follows, according to the results obtained.

[26] 1. ECH wave driven resonant scattering rates depend strongly on electron energy and equatorial pitch angle. Most intense pitch angle scattering by $\mathrm{ECH}$ waves occurs for $100 \mathrm{eV}-5 \mathrm{keV}$ plasma sheet electrons at a rate of $>10^{-4} \mathrm{~s}^{-1}$ and is well confined to $\alpha_{e q}<30^{\circ}$, similar to the previous results in the inner magnetosphere [e.g., Horne and Thorne, 2000; Thorne et al., 2010; Ni et al., 2011a].

[27] 2. Pitch angle scattering due to ECH emissions can approach or be comparable to the strong diffusion limit in the realistic magnetic field of the outer magnetosphere, producing efficient precipitation loss of plasma sheet electrons $<\sim 5 \mathrm{keV}$ on a loss timescale of a few hours or less. In contrast, ECH wave induced cross diffusion and momentum diffusion is rather inefficient.

[28] 3. The modeled intensity of the $557.7 \mathrm{~nm}$ green-line aurora, using the auroral electron transport model based upon the ionospheric precipitation flux inferred from $\mathrm{ECH}$ wave scattering rates, is consistent with the concurrent ground-based diffuse auroral observations and the estimated diffuse auroral intensity from the Maxwellian-type fitting to the energetic electron precipitation spectrum. This agreement confirms that the observed ECH wave activity, in the absence of whistler mode chorus waves, is responsible for the observed diffuse auroral emissions in the outer magnetosphere.

[29] While this investigation is a case study, a careful and feasible theoretical and numerical analysis has been carried out using the high-quality data and improved magnetic field model to reach a reasonable explanation of the conjugate observations in space and on ground. The results support the scenario that intensification of ECH emissions in the central plasma sheet can be strong enough to play an important or even dominant role in driving diffuse auroral precipitation in the outer magnetosphere. A recent THEMIS analysis of the global distribution of ECH waves by $\mathrm{Ni}$ et al. [2011c] has shown that moderately strong ( $>$ a few tens of $\mathrm{mV} / \mathrm{m}$ ) ECH waves on the nightside can be extended to beyond $\mathrm{L} \sim 12$, while nightside chorus waves have been found to be statistically very weak for L > 8 [Li et al., 2009a]. A number of recent studies based upon statistical wave information [Thorne et al., 2010; Ni et al., 2011a, 2011b] have found that the diffuse aurora at $\mathrm{L}<8$ is dominantly due to a combination of both upper and lower band chorus scattering. Our detailed event study implies that ECH waves tend to be a major wave mode associated with high-latitude diffuse 
auroral emissions and have the potential to modify the evolution of plasma sheet electrons and the global morphology of the diffuse aurora in the outer magnetosphere.

[30] ECH waves are driven by the electron loss cone distribution [e.g., Ashour-Abdalla and Kennel, 1978; Horne, 1989; Horne et al., 2003], and this mechanism still operates even when the loss cone is very small. In contrast, chorus excitation in both lower and upper band requires reasonably strong thermal anisotropy, which is only present inside $\mathrm{L} \sim 8$ on the nightside [ $\mathrm{Li}$ et al., 2010]. Chorus is generated by a nonlinear mechanism, thought to arise out of a band of weak noise from a temperature anisotropy. However, nonlinear theories of chorus generation [e.g., Omura et al., 2008, 2009; Omura and Nunn, 2011] show that it is generated very close to the magnetic equator and that there are special conditions for the waves to remain in resonance for nonlinear generation which depend on the field line gradient. These conditions may be more easily satisfied in the inner magnetosphere and more difficult at larger L-shells due to the stretching of the field line. The methodology developed in this study has the capability to quantitatively link the ground-based auroral observations with the magnetospheric source. Additional event analyses and theoretical investigations will be the subject of our future research to pursue an unambiguous and definitive understanding of the relationship between magnetospheric waves (electrostatic $\mathrm{ECH}$ emissions and electromagnetic chorus waves) and diffuse auroral precipitation on a global scale.

[31] Acknowledgments. This research was funded in part by the NSF grant ATM-0802843. We thank J. W. Bonnell for providing the THEMIS EFI data and U. Auster for the THEMIS FGM data. The research leading to these results has received funding from the European Community's Seventh Framework Programme (FP7/2007-2013) under grant agreement 262468. DL was supported by a subcontract of NASA grant NNX09AI04G to the University of Colorado.

[32] Masaki Fujimoto thanks the reviewers for their assistance in evaluating this paper.

\section{References}

Albert, J. M. (2007), Simple approximations of quasi-linear diffusion coefficients, J. Geophys. Res., 112, A12202, doi:10.1029/2007JA012551.

Albert, J. M., and Y. Y. Shprits (2009), Estimates of lifetimes against pitch angle diffusion, J. Atmos. Sol. Terr. Phys., 71, 1647-1652, doi:10.1016/j. jastp.2008.07.004.

Angelopoulos, V. (2008), The THEMIS mission, Space Sci. Rev., 141, 5-34, doi:10.1007/s11214-008-9336-1.

Ashour-Abdalla, M., and C. F. Kennel (1978), Nonconvective and convective electron cyclotron harmonic instabilities, J. Geophys. Res., 83 1531-1543, doi:10.1029/JA083iA04p01531.

Belmont, G., D. Fontaine, and P. Canu (1983), Are equatorial electron cyclotron waves responsible for diffuse auroral electron precipitation? J. Geophys. Res., 88, 9163-9170, doi:10.1029/JA088iA11p09163.

Bonnell, J. W., et al. (2008), The electric field instrument (EFI) for THEMIS, Space Sci. Rev., 141, 303-341, doi:10.1007/s11214-008-9469-2.

Chang, H. C. (1983), Cyclotron resonant scattering of energetic electrons by electromagnetic waves in the magnetosphere, Tech. Rep. E414-1, Space, Telecommun. and Radiosci. Lab., Stanford Univ., Stanford, Calif.

Coroniti, F. V. (1985), Space plasma turbulent dissipation: Reality or myth?, Space Sci. Rev., 42, 399-410, doi:10.1007/BF00214995.

Donovan, E. F. (1993), Modeling the magnetic effects of field-aligned currents, J. Geophys. Res., 98, 13,529-13,543, doi:10.1029/93JA00603.

Fontaine, D., and M. Blanc (1983), A theoretical approach to the morphology and the dynamics of diffuse auroral zones, J. Geophys. Res., 88, 7171-7184, doi:10.1029/JA088iA09p07171.

Glauert, S. A., and R. B. Horne (2005), Calculation of pitch-angle and energy diffusion coefficients with the PADIE code, J. Geophys. Res. 110, A04206, doi:10.1029/2004JA010851.
Gough, M. P., P. J. Christiansen, G. Martelli, and E. J. Gershuny (1979), Interaction of electrostatic waves with warm electrons at the geomagnetic equator, Nature, 279, 515-517, doi:10.1038/279515a0.

Hardy, D. A., M. S. Gussenhoven, and E. Holeman (1985), A statistical model of auroral electron precipitation, J. Geophys. Res., 90, 4229-4248, doi:10.1029/JA090iA05p04229.

Hardy, D. A., M. S. Gussenhoven, and D. Brautigam (1989), A statistical model of auroral ion precipitation, J. Geophys. Res., 94, 370-392, doi:10.1029/JA094iA01p00370.

Hedin, A. E. (1991), Extension of the MSIS thermosphere model into the middle and lower atmosphere, J. Geophys. Res., 96, 1159-1172, doi:10.1029/90JA02125.

Horne, R. B. (1989), Path-integrated growth of electrostatic waves: The generation of terrestrial myriametric radiation, J. Geophys. Res., 94, 8895-8909, doi:10.1029/JA094iA07p08895.

Horne, R. B., and R. M. Thorne (1993), On the preferred source location for the convective amplification of ion cyclotron waves, J. Geophys. Res., 98, 9233-9247, doi:10.1029/92JA02972.

Horne, R. B., and R. M. Thorne (1994), Convective instabilities of electromagnetic ion cyclotron waves in the outer magnetosphere, J. Geophys. Res., 99, 17,259-17,273, doi:10.1029/94JA01259.

Horne, R. B., and R. M. Thorne (2000), Electron pitch angle diffusion by electrostatic electron cyclotron harmonic waves: The origin of pancake distributions, J. Geophys. Res., 105, 5391-5402, doi:10.1029/ 1999JA900447.

Horne, R. B., P. J. Christiansen, M. P. Gough, K. G. Rönnmark, J. F. E. Johnson, J. Sojka, and G. L. Wrenn (1981), Amplitude variations of electron cyclotron harmonic waves, Nature, 294, 338-340, doi:10.1038/ 294338a0.

Horne, R. B., R. M. Thorne, N. P. Meredith, and R. R. Anderson (2003), Diffuse auroral electron scattering by electron cyclotron harmonic and whistler mode waves during an isolated substorm, J. Geophys. Res., 108(A7), 1290, doi:10.1029/2002JA009736.

Johnstone, A., D. Walton, R. Liu, and D. Hardy (1993), Pitch angle diffusion of low-energy electrons by whistler mode waves, J. Geophys. Res., 98, 5959-5967, doi:10.1029/92JA02376.

Kennel, C. F. (1969), Consequences of a magnetospheric plasma, Rev. Geophys., 7, 379-419.

Kennel, C. F., and H. Petschek (1966), Limit on stably trapped particle fluxes, J. Geophys. Res., 71, 1-28.

Kennel, C. F., F. L. Scarf, R. W. Fredricks, J. H. McGehee, and F. V. Coroniti (1970), VLF electric field observations in the magnetosphere, J. Geophys. Res., 75, 6136-6152, doi:10.1029/JA075i031p06136.

Kubyshkina, M., V. Sergeev, N. Tsyganenko, V. Angelopoulos, A. Runov, H. Singer, K. H. Glassmeier, H. U. Auster, and W. Baumjohann (2009), Toward adapted time-dependent magnetospheric models: A simple approach based on tuning the standard model, J. Geophys. Res., 114, A00C21, doi:10.1029/2008JA013547.

Kubyshkina, M., V. Sergeev, N. Tsyganenko, V. Angelopoulos, A. Runov, E. Donovan, H. Singer, U. Auster, and W. Baumjohann (2011), Timedependent magnetospheric configuration and breakup mapping during a substorm, J. Geophys. Res., 116, A00I27, doi:10.1029/2010JA015882.

Li, W., et al. (2009a), Global distribution of whistler-mode chorus waves observed on the THEMIS spacecraft, Geophys. Res. Lett., 36, L09104 doi:10.1029/2009GL037595.

Li, W., et al. (2009b), Evaluation of whistler-mode chorus intensification on the nightside during an injection event observed on the THEMIS spacecraft, J. Geophys. Res., 114, A00C14, doi:10.1029/2008JA013554.

Li, W., et al. (2010), THEMIS analysis of observed equatorial electron distributions responsible for the chorus excitation, J. Geophys. Res., 115, A00F11, doi:10.1029/2009JA014845.

Liang, J., W. W. Liu, E. Spanswick, and E. F. Donovan (2007), Azimuthal structures of substorm electron injection and their signatures in riometer observations, J. Geophys. Res., 112, A09209, doi:10.1029/2007JA012354.

Liang, J., V. Uritsky, E. Donovan, B. Ni, E. Spanswick, T. Trondsen, J. Bonnell, A. Roux, U. Auster, and D. Larson (2010), THEMIS observations of electron cyclotron harmonic emissions, ULF waves, and pulsating auroras, J. Geophys. Res., 115, A10235, doi:10.1029/2009JA015148.

Liang, J., B. Ni, E. Spanswick, M. Kubyshkina, E. F. Donovan, V. M. Uritsky, R. M. Thorne, and V. Angelopoulos (2011), Fast earthward flows, electron cyclotron harmonic waves, and diffuse auroras: Conjunctive observations and a synthesized scenario, J. Geophys. Res., 116 , A12220, doi:10.1029/2011JA017094.

Lui, A. T. Y., D. Venkatesan, C. D. Anger, S.-I. Akasofu, W. J. Heikkila J. D. Winningham, and J. R. Burrows (1977), Simultaneous observations of particle precipitations and auroral emissions by the Isis 2 satellite in the 19-24 MLT sector, J. Geophys. Res., 82, 2210-2226, doi:10.1029/ JA082i016p02210. 
Lummerzheim, D. (1987), Electron transport and optical emissions in the aurora, PhD thesis, Univ. of Alaska, Fairbanks.

Lyons, L. R. (1974), Electron diffusion driven by magnetospheric electrostatic waves, J. Geophys. Res., 79, 575-580, doi:10.1029/JA079i004p00575.

Meredith, N. P., R. B. Horne, A. D. Johnstone, and R. R. Anderson (2000), The temporal evolution of electron distributions and associated wave activity following substorm injections in the inner magnetosphere, J. Geophys. Res., 105, 12,907-12,917, doi:10.1029/2000JA900010.

Meredith, N. P., R. B. Horne, R. M. Thorne, and R. R. Anderson (2009), Survey of upper band chorus and ECH waves: Implications for the diffuse aurora, J. Geophys. Res., 114, A07218, doi:10.1029/2009JA014230.

Newell, P. T., T. Sotirelis, and S. Wing (2009), Diffuse, monoenergetic, and broadband aurora: The global precipitation budget, J. Geophys. Res., 114 A09207, doi:10.1029/2009JA014326.

Ni, B., R. M. Thorne, Y. Y. Shprits, and J. Bortnik (2008), Resonant scattering of plasma sheet electrons by whistler-mode chorus: Contribution to diffuse auroral precipitation, Geophys. Res. Lett., 35, L11106, doi:10.1029/ 2008GL034032.

Ni, B., R. M. Thorne, R. B. Horne, N. P. Meredith, Y. Y. Shprits, L. Chen, and W. Li (2011a), Resonant scattering of plasma sheet electrons leading to diffuse auroral precipitation: 1. Evaluation for electrostatic electron cyclotron harmonic waves, J. Geophys. Res., 116, A04218, doi:10.1029/ 2010JA016232

Ni, B., R. M. Thorne, N. P. Meredith, R. B. Horne, and Y. Y. Shprits (2011b), Resonant scattering of plasma sheet electrons leading to diffuse auroral precipitation: 2. Evaluation for whistler mode chorus waves, J. Geophys. Res., 116, A04219, doi:10.1029/2010JA016233.

$\mathrm{Ni}$, B., et al. (2011c), Global distribution of electrostatic electron cyclotron harmonic waves observed on THEMIS, Geophys. Res. Lett., 38, L17105, doi:10.1029/2011GL048793.

Ni, B., R. M. Thorne, Y. Y. Shprits, K. G. Orlova, and N. P. Meredith (2011d), Chorus-driven resonant scattering of diffuse auroral electrons in nondipolar magnetic fields, J. Geophys. Res., 116, A06225, doi:10.1029/ 2011JA016453.

Nishimura, Y., et al. (2011), Estimation of magnetic field mapping accuracy using the pulsating aurora-chorus connection, Geophys. Res. Lett., 38 , L14110, doi:10.1029/2011GL048281.

Omura, Y., and D. Nunn (2011), Triggering process of whistler mode chorus emissions in the magnetosphere, J. Geophys. Res., 116, A05205, doi:10.1029/2010JA016280.

Omura, Y., Y. Katoh, and D. Summers (2008), Theory and simulation of the generation of whistler-mode chorus, J. Geophys. Res., 113, A04223, doi:10.1029/2007JA012622.

Omura, Y., M. Hikishima, Y. Katoh, D. Summers, and S. Yagitani (2009), Nonlinear mechanisms of lower-band and upper-band VLF chorus emissions in the magnetosphere, J. Geophys. Res., 114, A07217, doi:10.1029/ 2009JA014206.
Orlova, K. G., and Y. Y. Shprits (2010), Dependence of pitch-angle scattering rates and loss timescales on the magnetic field model, Geophys. Res. Lett., 37, L05105, doi:10.1029/2009GL041639.

Roeder, J. L., and H. C. Koons (1989), A survey of electron cyclotron waves in the magnetosphere and the diffuse auroral electron precipitation, J. Geophys. Res., 94, 2529-2541, doi:10.1029/JA094iA03p02529.

Shprits, Y. Y., W. Li, and R. M. Thorne (2006), Controlling effect of the pitch-angle scattering rates near the edge of the loss cone on electron lifetimes, J. Geophys. Res., 111, A12206, doi:10.1029/2006JA011758.

Steele, D., and D. McEwen (1990), Electron auroral excitation efficiencies and intensity ratios, J. Geophys. Res., 95, 10,321-10,336, doi:10.1029/ JA095iA07p10321.

Summers, D., and B. Ni (2008), Effects of latitudinal distributions of particle density and wave power on cyclotron resonant diffusion rates of radiation belt electrons, Earth Planets Space, 60, 763-771.

Summers, D., B. Ni, and N. P. Meredith (2007a), Timescales for radiation belt electron acceleration and loss due to resonant wave-particle interactions: 1. Theory, J. Geophys. Res., 112, A04206, doi:10.1029/2006JA011801

Summers, D., B. Ni, and N. P. Meredith (2007b), Timescales for radiation belt electron acceleration and loss due to resonant wave-particle interactions: 2. Evaluation for VLF chorus, ELF hiss, and electromagnetic ion cyclotron waves, J. Geophys. Res., 112, A04207, doi:10.1029/2006JA011993.

Swift, D. W. (1981), Mechanisms for auroral precipitation: A review, Rev. Geophys., 19, 185-211, doi:10.1029/RG019i001p00185.

Tao, X., R. M. Thorne, W. Li, B. Ni, N. P. Meredith, and R. B. Horne (2011), Evolution of electron pitch angle distributions following injection from the plasma sheet, J. Geophys. Res., 116, A04229, doi:10.1029/ 2010JA016245

Thorne, R. M., B. Ni, X. Tao, R. B. Horne, and N. P. Meredith (2010), Scattering by chorus waves as the dominant cause of diffuse auroral precipitation, Nature, 467, 943-946, doi:10.1038/nature09467.

Tsyganenko, N. A. (1989), A magnetospheric magnetic field model with a warped tail current sheet, Planet. Space Sci., 37, 5-20, doi:10.1016/00320633(89)90066-4

V. Angelopoulos, Institute of Geophysics and Planetary Physics, University of California, Los Angeles, CA 90095, USA.

E. F. Donovan, J. Liang, and E. Spanswick, Department of Physics and Astronomy, University of Calgary, Calgary, AB T2N 1N4, Canada.

R. B. Horne, British Antarctic Survey, Madingley Road, Cambridge CB3 OET, UK

M. Kubyshkina, Institute of Physics, Saint Petersburg State University, St. Petersburg 198504, Russia.

D. Lummerzheim, Geophysical Institute, University of Alaska Fairbanks, Fairbanks, AK 99775, USA.

B. Ni and R. M. Thorne, Department of Atmospheric and Oceanic Sciences, University of California, Los Angeles, CA 90095, USA. (bbni@atmos.ucla. edu) 